\title{
Acetaminophen pre-treatment for Closed Nose Reduction under local anesthesia: a triple-blind placebo-controlled randomized clinical trial
}

\author{
Saeed Sohrabpour ${ }^{1}$, Mozhgan Safaeyan ${ }^{2}$, Keyvan Aghazadeh ${ }^{1}$, Mohammadreza Firouzifar $^{1}$, \\ and Benyamin Mousavi-asl ${ }^{1}$ \\ ${ }^{1}$ Tehran University of Medical Sciences \\ ${ }^{2}$ Islamic Azad University Tehran Medical Sciences
}

July 7,2020

\begin{abstract}
Background: Nasal bone fracture is the most common facial fracture (approximately $40 \%$ of all the facial fractures) and is mostly treated with closed reduction. It is performed either with general or local anesthesia. Local anesthesia is fast and cheap but pain can't be fully suppressed. We designed this study to see if pre-emptive analgesia with Acetaminophen can reduce the pain associated with nasal reduction under local anesthesia. Methodology/Principal: in this triple-blind randomized clinical trial we had 100 participants divided into two arms (either placebo or Acetaminophen $500 \mathrm{mg}$ Tablet 45 minutes before reduction). Medication was randomly delivered via packages and the surgeon, patients, and data analyzer were all unaware of the nature of the medication. Then demographic data along with VAS pain scores (1-10 score from least to most severe pain) during local anesthesia, during reduction, and 24 hours after reduction were analyzed. Results: The patients were mostly men (74\%) and the most common mechanism was involvement in fight. (30\%). Left depression was the most seen type of fracture (53\%). Pain scores as well as surgeon satisfaction analysis returned no statistical difference between the two groups. Correlation analysis was done and the only factor for pain severity during reduction was the number of tries needed. Conclusions: Acetaminophen pre-treatment can't add analgesia any more than that of Placebo. Its use before reduction of nasal bone is not justified
\end{abstract}

\section{Abstract:}

Introduction: Nasal bone fracture is the most common facial fracture (approximately $40 \%$ of all the facial fractures) and is mostly treated with closed reduction. It is performed either with general or local anesthesia. Local anesthesia is fast and cheap but pain can't be fully suppressed. We designed this study to see if preemptive analgesia with Acetaminophen can reduce the pain associated with nasal reduction under local anesthesia.

Methods: in this triple-blind randomized clinical trial we had 100 participants divided into two arms (either placebo or Acetaminophen $500 \mathrm{mg}$ Tablet 45 minutes before reduction). Medication were randomly delivered via packages and the surgeon, patients and data analyzer were all unaware of the nature of medication. Then demographic data along with VAS pain scores (1-10 score from least to most severe pain) during local anesthesia, during reduction and 24 hours after reduction were analyzed.

Result: the patients were mostly men $(74 \%)$ and the most common mechanism was involvement in fight. (30\%). Left depression was the mostly seen type of fracture (53\%). Pain scores as well as surgeon satisfaction analysis returned no statistical difference between the two groups. Correlation analysis was done and the only factor for pain severity during reduction was the number of tries needed. 
Conclusion: Acetaminophen pre-treatment can't add analgesia any more than that of Placebo. Its use before reduction of nasal bone is not justified.

Keywords: Nasal bone fracture, closed reduction, Acetaminophen, analgesia, pre-emptive

Keypoints:

1. Acetaminophen pretreatment is not justified before closed nose reduction

2. Success rate of nose reduction under local anesthesia is comparable to that of general anesthesia

3. Pain scores only correlated with number of tries for reduction

4. Pain scores do not correlate with sex, age or time elapsed from fracture .

5. Pain scores can be different in different ethnic/cultural groups.

Introduction:

Nasal bone fracture (NBF) is the most common fracture of the facial skeleton (approximately $40 \%$ of all the facial fractures) and is ranked third in fractures throughout the body. The final goal of treatment in NBF is to return the nose to its pre-fracture state, both aesthetically and functionally. ${ }^{1}$ This relies mostly on the mechanism of fracture and severity of the injury. The treatment is either closed or open reduction. Open reduction is reserved for more severe injuries (e.g. septal fracture, commuted fracture, open fracture and coexisting fracture in other parts of the facial skeleton). Closed reduction is the most common way of treatment and it can be done under general or local anesthesia. Local anesthesia is less expensive, faster and has not shown to alter the effectiveness of reduction ${ }^{2-4}$. The limiting factor is the pain during the procedure which can't be entirely controlled. Some physicians use pre-emptive analgesia to increase patient satisfaction and decrease the pain burden. ${ }^{5,6}$ Using this concept, we wanted to assess the effectiveness of oral acetaminophen - a known and fairly safe medicine in dealing with pains related to bone fracture ${ }^{7,8_{-}}$in patients who undergo closed nasal reduction under local anesthesia.

Methods:

In this triple-blind randomized clinical trial, a total of 105 patients were selected based on inclusion/exclusion criteria. The study setting was a "nose fracture" clinic in a tertiary referral center. (-). Patients were 18 years of age or older and written consent was obtained from all of them at the time of inclusion. Those with open and/or commuted fracture, septal fracture, cartilaginous damage, and concomitant maxillofacial fractures were excluded because these require open reduction. We also excluded any patient with hepatic disease. A questionnaire was filled comprised of age, sex, duration between fracture and reduction and mechanism of fracture for 105 patients. Patients were coded randomly using a random digits table. Each code received a package in a sequence consisted of either Acetaminophen $500 \mathrm{mg}$ tablet(HAKIM Pharmaceutical Co. Tehran, Iran) or Placebo which was administered orally 45 minutes before the time of reduction. The two groups were designed to be parallel and allocation ratio was 1:1. The surgeon and the patients and the data collector were unaware of the nature of the medications. Letters A and B were used as group names in the analysis to ensure blindness for the data analyzer. Only after the analysis was done the true nature of each group was revealed. all the Patients underwent local anesthesia using ethmoidal and infraorbital nerve blocks with $2 \%$ lidocaine injection then xylocaine $+1 / 100000$ adrenalin soaked pledgets were placed inferior to the nasal bone to anesthetize the mucosa. The nasal reduction was performed using bone elevator for all the patients. Pain during anesthesia and during reduction was recorded using visual analog scale (VAS) . The scale comprised of 10 points in which 1 was the least and 10 was the most severe amount of pain. The surgeon satisfaction both aesthetically and functionally (analog scale 1-10), number of tries to achieve reduction and need for packing to stop bleeding were also recorded. Patients were contacted 24 hours after reduction and the VAS was filled for each one. Then data were analyzed using IBM SPSS Statistics for Windows (ver. 22). Demographic Data were analyzed using chi-square and descriptive analysis. Pain comparison in two groups was analyzed using non-parametric Mann-Whitney test. Bivariate correlation analysis was also done. This study was approved by the ethics committee of - .

Results: 
105 patients agreed (52 in intervention and 53 in placebo group) to participate but 100 completed the study. 49 patients received Acetaminophen and 51 received Placebo. Chi-square analysis revealed that patients were mostly men and the two groups were fairly similar in sex distribution $(\mathrm{p}=0.821)$ (Table- 1$)$. The damage mechanism (Table-2) in the two groups was not statistically different although there were more dorsum depressions in the Acetaminophen group (Table-3, $\mathrm{p}=0.016$ ) and swelling was greater in the placebo group (Table-4, $\mathrm{p}=0.012$ ). Mean $\pm \mathrm{SD}$ age was $33.09 \pm 14.66$ (Placebo) and $28.81 \pm 11.58$ (Acetaminophen) years $(\mathrm{p}=0.109)$ and Mean \pm SD time to reduction was $5.03 \pm 2.3$ (Placebo) and 6.08 \pm 3.4 (Acetaminophen) days $(\mathrm{p}=0.052)$.

No specific side effects were documented in the two groups. Pain score (during anesthesia, during reduction and 24 hours after reduction), surgeon satisfaction and the number of tries per reduction did not follow a normal distribution (using K-S test) so we used none parametric Mann-Whitney test to compare two groups. The results are summarized in Table-5. The two groups were not statistically significant.(Figure -1)

Taking placebo group into account, there was also no significant correlation between pain during reduction and $\operatorname{sex}(\mathrm{p}=0.797)$, trauma mechanism $(\mathrm{p}=0.199)$, swelling $(\mathrm{p}=0.366)$, type of damage $(\mathrm{p}=0.822)$, $\operatorname{age}(\mathrm{p}=0.875)$,time elapsed from the $\operatorname{injury}(\mathrm{p}=0.504)$ and need to stop the bleeding $(\mathrm{p}=0.230)$. It only correlated with number of tries per reduction $(\mathrm{p}=0.015)$. (Table-6)

\section{Discussion:}

This was a triple-blind randomized clinical trial in which we wanted to measure the effect of Pre-emptive analgesia with Acetaminophen on pain in the closed nose reduction as well as the result of the reduction. To our knowledge, it is the first randomized clinical trial to assess pre-emptive analgesia in closed nasal reduction. Because pain is a subjective variable we used visual analog scale (VAS) to measure it in our study. We observed similar efficacy between Acetaminophen and placebo in pain control using VAS during local anesthesia, during reduction and 24 hours after the reduction. Our patients were mostly men which is in agreement with prior studies ${ }^{2-4,9}$ we also observed that the pain during reduction does not correlate with gender, trauma mechanism, swelling severity, type of damage and the time elapsed since the injury and the only thing that correlated was the number of tries per each reduction. This is in contrast with Fernando Vilela's study in which they state that pain correlates with time elapsed since the reduction. Their sample size was smaller and their study design was a longitudinal cohort ${ }^{2}$. The mean score of pain during the reduction in our study was higher compared to a recent study which could be because of ethnic and cultural differences. We also observed that all but one patient didn't have significant pain (score 1 or 2 out of 10) $24 \mathrm{~h}$ after the procedure which is in contrast with this study in which they reported median score of 3 for music therapy and 5 in their control group in 1-week postoperative time.$^{10}$ we also observed high surgeon satisfaction score in both groups and overall which underlines the fact that local anesthesia can produce good results which are comparable with general anesthesia. ${ }^{2-4}$ the fact that we carefully designed this study to be triple-blind, makes our results fairly reliable. Some of patients didn't cooperate after the reduction so they were excluded and the fact that they may have had any other medication to ease their pain when in home may make a possible bias. We propose further evaluation of different medication and local anesthesia techniques to find better ways of reducing pain during nasal reduction.

Conclusion:

Acetaminophen pretreatment does not add more anesthesia other than that of Placebo. So it's prescription before nose reduction is not justified.

Acknowledgment:

We hereby thank all of the patients who participated in this study.

Conflict of Interest: NONE

Declaration because of U.S. Sanctions : All the authors do research and work in university and none work for other agencies. None of the authors is a representative of government in any way. 


\section{References:}

1. Hoffmann JF. An Algorithm for the Initial Management of Nasal Trauma.Facial Plast Surg . 2015;31(3):183-193. doi:10.1055/s-0035-1555618

2. Vilela F, Granjeiro R, Maurício C, Andrade P. Applicability and Effectiveness of Closed Reduction of Nasal Fractures under Local Anesthesia. Int Arch Otorhinolaryngol . 2014;18(3):266-271. doi:10.1055/s0034-1368138

3. Khwaja S, Pahade A V, Luff D, Green MW, Green KMJ. Nasal fracture reduction: local versus general anaesthesia. Rhinology . 2007;45(1):83-88. http://www.ncbi.nlm.nih.gov/pubmed/17432077. Accessed March $4,2020$.

4. Atighechi S, Baradaranfar MH, Akbari SA. Reduction of nasal bone fractures: A comparative study of general, local, and topical anesthesia techniques. J Craniofac Surg . 2009;20(2):382-384. doi:10.1097/SCS.0b013e31819b945f

5. Skinner HB. Multimodal acute pain management. Am J Orthop (Belle Mead NJ) . 2004;33(5 Suppl):5-9.

6. Hoard MA, Bill TJ, Campbell RL. Reduction in morbidity after iliac crest bone harvesting: The concept of preemptive analgesia. J Craniofac Surg . 1998;9(5):448-451. doi:10.1097/00001665-199809000-00011

7. Gao T, Zhu H, Zhang C, et al. Efficacy of acetaminophen with and without oxycodone for analgesia in non-operative treatment of extremity fractures in adults: Protocol for a double-blind randomized clinical trial. Trials . 2019;20(1):510. doi:10.1186/s13063-019-3579-x

8. Furyk J, Levas D, Close B, et al. Intravenous versus oral paracetamol for acute pain in adults in the emergency department setting: A prospective, double-blind, double-dummy, randomised controlled trial.Emerg Med J . 2018;35(3):163-168. doi:10.1136/emermed-2017-206787

9. Sung JY, Kang KD, Kim MW, Kim JH. Effect of lidocaine on pain caused by nasal pack removal after closed reduction of nasal bone fractures. Arch Plast Surg . 2020;47(1):15-19. doi:10.5999/aps.2019.00913

10. Ortega A, Gauna F, Munoz D, Oberreuter G, Breinbauer HA, Carrasco L. Music Therapy for Pain and Anxiety Management in Nasal Bone Fracture Reduction: Randomized Controlled Clinical Trial. Otolaryngol - Head Neck Surg (United States) . 2019;161(4):613-619. doi:10.1177/0194599819856604

\section{Hosted file}

tables.docx available at https://authorea.com/users/339328/articles/465578-acetaminophenpre-treatment-for-closed-nose-reduction-under-local-anesthesia-a-triple-blind-placebocontrolled-randomized-clinical-trial 